\title{
¿Hay matemáticas fuera de la escuela?: reflexiones de maestros de matemáticas en ejercicio ${ }^{1}$
}

\section{Is there math outside of school: math teacher reflections on exercise}

\author{
Existe matemática fora da escola: professor de \\ matemática reflexões sobre o exercício
}

Recibido: mayo 2013

Aceptado: agosto 2013
María Luisa Oliveras ${ }^{2}$

Hilbert Blanco-Álvarez ${ }^{3}$

Pedro Palhares ${ }^{4}$

\begin{abstract}
Resumen
Esta comunicación presenta los avances de una investigación ${ }^{5}$ cuya primera parte tiene como objetivo analizar las convergencias y tensiones de los maestros a la hora de reconocer como matemáticas saberes extraescolares o rechazarlos. El material empírico procede de un programa de formación de maestros en ejercicio en Etnomatemática realizado en Tumaco-Colombia entre julio y octubre de 2012, y está compuesto por reflexiones orales de los maestros registradas en grabaciones de audio. El marco teórico usado para el análisis de los datos ha sido la Etnomatemática de D’Ambrosio y la filosofía de las matemáticas de Wittgenstein.
\end{abstract}

Palabras clave: Etnomatemática; profesor; formación continuada de maestros; currículo cultural: diversidad; enseñanza; metodología de enseñanza; análisis y reflexión sobre la enseñanza.

\begin{abstract}
This paper presents the advances of a research1 the first part is to analyze the convergences and tensions when teachers recognize as mathematics or reject curricular knowledge. The empirical material comes from a teacher training program in Ethnomathematics exercise conducted in Tumaco, Colombia between July and October 2012, and consists of oral reflections registered teachers in audio. The theoretical framework used for the analysis of the data has been D'Ambrosio Ethnomathematics and philosophy of mathematics from Wittgenstein.
\end{abstract}

Keywords: Ethnomathematics, teacher, continuing education of teachers, curriculum cultural diversity, education, teaching methodology, analysis and reflection on teaching.

\footnotetext{
Artículo de Investigación.

Universidad de Granada, España. Contacto: oliveras@ugr.es

Universidad de Nariño, Colombia y Estudiante de Doctorado Universidad de Granada, España. Contacto: hilbla@yahoo.com

Universidade do Minho, Portugal. Contacto: palhares2307@gmail.com

La parte empírica de esta investigación ha sido patrocinada por la Fundación Save the Children International y ha contado con la participación del Departamento de Matemáticas y Estadística de la Universidad de Nariño, Pasto, Colombia.
} 


\section{Resumo}

Este artigo apresenta os avanços de um investigaçãol a primeira parte é analisar as convergências e tensões quando os professores reconhecem como matemática ou rejeitar o conhecimento curricular. $\mathrm{O}$ material empírico trata de um programa de formação de professores em Etnomatemática exercício realizado em Tumaco, na Colômbia, entre julho e outubro de 2012, e consiste em reflexões orais professores registradas em áudio. O referencial teórico utilizado para a análise dos dados foi D’Ambrosio Etnomatemática e filosofia da matemática de Wittgenstein.

Palavras-chave: Etnomatemática, professor, educação continuada de professores, diversidade cultural currículo, educação, metodologia de ensino, análise e reflexão sobre o ensino.

\section{Introducción}

Esta comunicación presenta avances de la primera parte la investigación doctoral "La formación de maestros de matemáticas y la Etnomatemática: hacia un modelo didáctico" dirigida por la Dra. María Luisa Oliveras al interior del Grupo de Investigación Etnomatemáticas, Formación de profesores y Didáctica de la Universidad de Granada, España.

El objetivo general de la investigación es presentar elementos para el diseño de un modelo didáctico que pueda servir de referencia para la formación de maestros de matemáticas en ejercicio orientado desde la Etnomatemática. En esta comunicación solo se presentan los datos y el análisis referido al objetivo específico de analizar las convergencias y tensiones de los maestros a la hora de reconocer, o no, saberes extraescolares como matemáticas. Tensiones que son reconocidas en distintas investigaciones por investigadores nacionales como Jaramillo (2011), Tamayo \& Jaramillo (2011), Aroca (2010) y Blanco-Álvarez (2011).

En el ámbito internacional, existe un amplio número de publicaciones y de investigaciones referidas a las tensiones entre la matemática extraescolar y las matemáticas académicas (Oliveras, 1996, 2005, 2006; Oliveras \& Gavarrete, 2012; Santillán, A. \& Zachman, 2009; Knijnik \& Meregalli, 2012; entre otros).

\section{Marco teórico}

El marco teórico utilizado en esta primera parte de la investigación fueron, por un lado, las reflexiones acerca de la Etnomatemática de D’Ambrosio (1997). Éste hace una diferenciación entre las matemáticas que son enseñadas en la escuela y que llama "matemáticas académicas" en contraposición a la Etnomatemática que define como "las matemáticas que se practica entre grupos culturales identificables, tales como sociedades de tribus nacionales, grupos laborales, niños de cierto rango de edades, clases profesionales, entre otros" (p. 16), teniendo en cuenta "(...) las capacidades de clasificar, ordenar, inferir y modelar" (p. 17) y que, generalmente, son aprendidas en contextos extraescolares.

Por otro lado, se hace uso de las reflexiones sobre la filosofía de las matemáticas que Wittgenstein (1999) presenta en Investigaciones filosóficas, donde "establece otra teoría del significado basada en el uso público del lenguaje y en la noción de 'juego lingüístico', lo que indica su cambio hacia una concepción del mundo y del proceso cognoscitivo interactiva o epistemológicamente transaccional" (Oliveras, 1996, p. 68), y donde "cada lenguaje es un modo de conocer que ha desarrollado un grupo de seres humanos" (Ibid., p. 74). En este mismo sentido, Knijnik (2007, p. 75) señala que "Wittgenstein ayuda a fundamentar filosóficamente el campo de la Etnomatemática, especialmente en lo que dice respecto a la no existencia de una sola matemática, esa 
que llamamos "la" Matemática, asociada a la racionalidad moderna (...)”.

Los conceptos de Wittgenstein, de acuerdo a Knijnik (2012) que apoyan la investigación Etnomatemática son: juegos de lenguaje que hacen relación a los distintos lenguajes o formas de expresar diferentes procesos matemáticos como construcción de hipótesis, resolución de problemas, etc. Lenguajes que hacen parte de formas de vida en las cuales son cargados de significados culturales. Finalmente, los juegos de lenguaje no están separados, así pertenezcan a diferentes formas de vida pues éstos gozan de semejanzas de familia en tanto que hacen parte de una complicada red de similitudes y entrecruces.

\section{Diseño de investigación}

Aproximación metodológica. Esta parte de la investigación se centra en un Estudio de Caso de carácter interpretativo, siguiendo una aproximación etnográfica. El caso es un programa de formación en Etnomatemática para maestros en ejercicio realizado en el municipio de Tumaco, Colombia entre julio y octubre de 2012.

Instrumentos de recolección de datos. En esta parte de la investigación se consideró adecuado recoger la información por medio de grabaciones de audio.

Población. El grupo que participó en la investigación fue de 23 maestros de la educación básica primaria, y 5 de la educación básica secundaria, del municipio de Tumaco, Colombia. Todos ellos tienen a cargo el área de matemáticas y su formación profesional es muy diversa, lo cual enriqueció las discusiones y las perspectivas frente a la enseñanza y el aprendizaje de las matemáticas. Finalmente, es importante señalar que los maestros participantes se inscribieron al programa de formación de manera voluntaria.

Análisis de los datos. El material empírico analizado fueron las grabaciones de audio de las sesiones donde los maestros, organizados en grupos, daban sus opiniones sobre una lista de 18 frases que fueron tomadas de Blanco-Álvarez (2012) y que él utilizó para indagar sobre las actitudes hacia una postura sociocultural y política de la educación matemática de maestros en formación inicial. Las grabaciones de audio fueron transcriptas y se analizaron a la luz de la perspectiva Etnomatemática de D’Ambrosio (1997) y la filosofía de las matemáticas de Wittgenstein (1996). El proceso de análisis se apoyó en el uso del software Atlas.ti.

\section{Resultados}

De acuerdo con el marco teórico señalado, el material empírico fue examinado con el fin de buscar puntos de convergencia y tensiones de los maestros a la hora de reconocer, o no, saberes extraescolares como matemáticas.

Un resultado llamativo fueron las reflexiones presentadas por los maestros sobre la frase: "Las mujeres africanas que tejen figuras como cuadrados, círculos, triángulos, etc. en los cestos o en la ropa tienen nociones de geometría".

M1: Yo pienso que ellos no tienen nociones de geometría porque ellos la palabra ni la conocen, geometría, de pronto la figura de una forma empírica la pueden elaborar pero no saben ni siquiera las medidas, o sea, no saben cómo se elabora de una forma científica sino, de pronto de una forma empírica la han aprendido a hacer, ellas saben hacerla, pero si tú le dices hágame una circunferencia de tanto de diámetro y de radio ellos no lo van a saber hacer.

Esta respuesta, desde la perspectiva de D'ambrosio (1997) permite ver cómo el maestro reconoce la existencia de una matemática académica, y todo aquel conocimiento que no goce del formalismo de éstas no es entendido como tal. En palabras de Wittgenstein (1996) puede interpretarse como el no reconocimiento, por parte del maestro, de semejanzas de familia entre los juegos de lenguaje de la matemática académica y los juegos de lenguaje de las mujeres tejedoras de África. Puesto que el primero tiene una forma de nombrar los objetos matemáticos y de expresar sus propiedades, mientras que el segundo no. 
M2: $\quad$ Yo considero que ellas si sabían la geometría, yo creo que en cada etapa de la vida sabemos utilizar esos términos porque la geometría es un término científico, pero en diferentes culturas manejan otros términos de palabras y siempre lo que se ha cambiado en las diferentes etapas de la vida son los términos, nosotros sabemos que nos metieron en los términos estándares, lo que ha cambiado son los términos, pero sí se ha sabido qué manejo le han dado a ese concepto.

Este maestro, al contrario del anterior, reconoce la semejanza de familia existente entre los juegos de lenguaje de la matemática académica, a los que él se refiere como "científico" o "los términos estándares" y los juegos de lenguaje de las mujeres tejedoras de África, con su propia forma de vida, que hacen uso de diferentes términos en su cultura para referirse a la geometría.

Otro resultado sugestivo fueron las reflexiones presentadas por los maestros sobre la frase: "Fuera de la escuela no se aprenden matemáticas".

M3: $\quad$ Pero mire que muchas veces nosotros los profesores o padres de familia, cuando uno saca el niño al tablero, y dice: no, es que ese niño no sabe sumar ni sabe restar; mire, perdió porque ese niño no sabe sumar ni restar, pero tenemos en cuenta solo el concepto de acá (escuela), pero cuando uno lo manda a la tienda con un billete de $\$ 5000$ (pesos colombianos), el niño no se deja robar, el niño sabe sumar, sabe restar y tomamos en cuenta lo de acá y no lo de afuera donde el niño se desenvuelve. A la hora del recreo, cuando va a comprar a la tienda, dice aquí me falta.

M4: Los niños que van a "conchar" al mar, los niños son expertos contando cuántas conchas hay y cuentan por pares o de 5 en 5 y eso es una facilidad con la que cuentan y yo como profesora ya me es difícil hacerlo.

Estas maestras, reconocen la existencia de juegos de lenguaje en formas de vida distintas a la forma de vida escolar y reflexionan sobre la poca o ninguna atención que a estos juegos del lenguaje se le presta en la escuela y sobre la habilidad de los niños de cálculo mental por fuera del aula

En este sentido Knijnik (2007) señala:

Él (Wittgenstein) nos permite indagar sobre nuestras pedagogías escolares, que, si por ventura incluyen la enseñanza de "los cálculos mentales”, lo hace sin considerar como referencial los modos de operar de "las gentes de la calle", esas personas que en nada o muy poco estuvieron sometidas a los procesos de escolarización formal (p.77)

\section{Conclusiones}

Las evidencias obtenidas en la primera fase de la investigación señalan las convergencias de las ideas de los maestros hacia los juegos de lenguaje, en tanto que varios de ellos reconocen la existencia de pensamiento matemático extraescolar, pero también son evidentes las tensiones existentes ante reconocer semejanzas de familia entre los saberes extraescolares y los juegos de lenguaje de la matemática académica.

Por otro lado, se han mostrado ejemplos en los cuales los maestros no solo reconocen pensamiento matemático extraescolar en los niños, sino que además son conscientes de no tener en cuenta esto en el aula de clase, lo que conlleva a una ruptura entre la escuela y el entorno cotidiano del estudiante.

La conclusión anterior debe ubicarse dentro de una reflexión más general, en relación a cómo incorporar los saberes matemáticos extraescolares al currículo escolar, qué tipo de actividades diseñar y el papel de la comunidad en el aula de clase.

\section{Referencias}

Aroca, A. (2010). Una experiencia de formación docente en Etnomatemáticas: estudiantes afrodescendientes del Puerto de 
Buenaventura, Colombia. Revista Horizontes, 28(1), 87-95.

Blanco-Álvarez, H. (2012). Estudio de las actitudes hacia una postura sociocultural y política de la Educación Matemática en maestros en formación inicial. REDIMAT - Journal of Research in Mathematics Education, 1(1), 57-78.

Blanco-Álvarez, H. (2011). La postura sociocultural de la educación matemática y sus implicaciones en la escuela. Revista Educación y Pedagogía, 23(59), 59-66.

D'Ambrosio, U. (1997). Ethnomathematics and its place in the history and pedagogy of mathematics. En A. Powell \& M. Frankenstein. (eds.), Ethnomathematics: Challenging Eurocentrism in Mathematics Education (pp. 13-24). Albany: State University of New York.

Jaramillo, D. (2011). La educación matemática en una perspectiva sociocultural: tensiones, utopías, futuros posibles Revista Educación y Pedagogía, 23(59), 13-36.

Knijnik, G. (2012). Differentially positioned language games: ethnomathematics from a philosophical perspective. Educational Studies in Mathematics. 80, 87-100.

Knijnik, G. (2007). Diversidad cultural, matemáticas y exclusión: oralidad y escrita en la educación matemática campesina del sur del Brasil. En J. Giménez, J. Díez-Palomar, M. Civil (coords.), Educación matemática y exclusión (pp. 66-83). Barcelona: Editorial Graó.

Knijnik, G. \& Meregalli, J. (2012). Educação matemática em cursos de pedagogia: um estudo com professores dos anos iniciais do ensino fundamental. Revista Latinoamericana de Etnomatemática, 5(2). 4-20.

Oliveras, M., L. (1996). Etnomatemática, formación de profesores e innovación curricular maestros e interculturalidad. Granada: Editorial Comares.

Oliveras, M. L. (2005). Microproyectos para la educación intercultural en Europa. Uno: Revista de Didáctica de las Matemáticas, 38, 70-81.

Oliveras, M. L. (2006). Etnomatemáticas. De la multiculturalidad al mestizaje. En: J. M. Goñi (coord.), Matemáticas e interculturalidad (pp. 117-149). Barcelona: Editorial Grao.

Oliveras, M. L. \& Gavarrete, M. L. (2012). Modelo de aplicación de etnomatemáticas en la formación de profesores para contextos indígenas en Costa Rica. RELIME. Revista latinoamericana de investigación en matemática educativa, 15(3), 339-372.

Santillán, A. \& Zachman, P. (2009). Una experiencia de capacitación en Etnomatemática. Revista Latinoamericana de Etnomatemática, 2(1), 27-42.

Tamayo, C. \& Jaramillo, D. (2011). (Re)significación del currículo escolar indígena, relativo al conocimiento matemático, desde y para las prácticas sociales: el caso de la Comunidad Tile de Alto Caimán. En Memorias $12^{\circ}$ Encuentro Colombiano de Matemática Educativa. p. 865 -875 .

Wittgenstein, L. (1999). Investigaciones filosóficas. Barcelona: Ediciones Altaya 